\title{
Experimental techniques for study of chromatin mechanics in intact nuclei and living cells
}

\author{
Valerie L. R. M. Verstraeten ${ }^{1,2,3}$ \& Jan Lammerding ${ }^{1 *}$ \\ ${ }^{1}$ Department of Medicine, Cardiovascular Division, Brigham \& Women's Hospital/Harvard Medical School, \\ Boston, MA, USA; Tel: +1-617-768-8273; Fax: +1-617-768-8280; E-mail: jlammerding@rics.bwh.harvard.edu; \\ ${ }^{2}$ Department of Dermatology, University Hospital Maastricht, Maastricht, The Netherlands; ${ }^{3}$ School \\ for Oncology and Developmental Biology (GROW), Maastricht University, Maastricht, The Netherlands \\ * Correspondence
}

Key words: atomic force microscopy, cellular strain, chromatin, fluorescence image microdeformation, micropipette aspiration, nuclear mechanics

\begin{abstract}
While the structure of chromatin and its physical properties have been well studied on isolated chromatin fibres and DNA strands in vitro, its organization and function in the intact interphase nucleus is less clear. Chromatin organization is critical for transcriptional regulation and DNA replication, and mounting evidence suggests that cells respond to changes in the mechanical environment with alterations in nuclear architecture that are accompanied by modifications in gene expression. Since the nucleus forms part of a continuous physical network spanning the extracellular matrix, the cytoskeleton and the nuclear envelope, environmentally mediated forces can be transmitted to the nucleus and induce deformations of the chromatin.

Here, we describe a subset of techniques that can be applied to probe nuclear mechanics, ranging from micropipette aspiration to strain experiments on living cells. These experiments probe the physical properties of the nuclear envelope, the nucleoplasm, and the chromatin. We discuss the advantages and disadvantages of each technique and elaborate on their use to examine specific aspects of chromatin. In the end, a combination of these technologies can provide important insights into the delicate relationship between form and function of chromatin organization in the living cell.
\end{abstract}

\section{Introduction}

Structural organization of chromatin in the interphase nucleus

Cells exhibit the plasticity necessary to modulate their gene expression pattern during development. These alterations in gene expression often coincide with distinct changes in chromatin structure. The precise organization of chromatin within the eukaryotic nucleus remains a subject of debate, although there is consensus about its basic structure. Chromatin fibres are about $30 \mathrm{~nm}$ in diameter and exhibit a helical structure that is based on a periodic wrapping of DNA around histone octamers. Different models of helical chromatin wrapping have been proposed, but it is generally believed that (1) the nucleosomes are peripherally located in the chromatin fibre allowing a zig-zag chain structure at low ionic strength; (2) the linker histone which is essential for chromatin compaction is situated in the fibre interior; and (3) nucleosome-nucleosome interactions may 
drive the compaction and thereby contribute to the stability of the chromatin fibre (van Holde \& Zlatanova 2007). Chromatin fibres are further organized in higher-order chromatin domains such as highly compacted heterochromatin, chromatin associated with a multitude of nuclear bodies such as the nucleolus, and more loosely packed euchromatin. Whereas heterochromatin is mostly transcriptionally silent and located at the nuclear periphery, euchromatin constitutes gene-rich chromosome regions localized towards the centre of the nucleus and at nuclear pores. An additional layer of complexity evolves from the nonrandom spatial organization of the genome itself, in which each chromosome occupies a specific chromosome territory within the interphase cell nucleus with a preferential position relative to the centre of the nucleus and to the other chromosomes (reviewed in Parada \& Misteli 2002, Misteli 2004). The functional significance of spatial positioning regarding gene regulation and genome stability remains poorly understood, however.

\section{Chromatin and the nuclear envelope}

Recent data suggest that the nuclear envelope can contribute to chromosomal positioning by anchoring specific chromosomal regions at the nuclear periphery (Malhas et al. 2007). The nuclear envelope consists of a lipid double-membrane layer (i.e. the inner and outer nuclear membranes) and a filamentous network (i.e. the nuclear lamina) lining the inner side of the nuclear envelope. Nuclear lamins are the major building blocks of the lamina and are essential in chromatin scaffolding and maintenance of genomic integrity (reviewed in Verstraeten et al. 2007). In particular, studies in Drosophila melanogaster have indicated that nuclear lamins preferentially bind to large intergenic regions of transcriptionally inactive chromatin with mid- to late replication timing and lack of active histone modifications (Pickersgill et al. 2006). Lamins can also affect chromatin organization indirectly through their interaction with nuclear membrane-associated proteins that share a conserved LEM-domain which mediates their interaction with the DNA-crosslinking protein Barrier to Auto-integration Factor (BAF) (Laguri et al. 2001). Aside from the LEM-domain containing proteins, lamins interact with a multitude of other proteins that are thought to provide chromatin-anchorage sites at the inner nuclear membrane, e.g. the lamin B receptor (Worman et al. 1990, Gotzmann \& Foisner 1999), or affect gene regulation (Verstraeten et al. 2007).

Force transmission between the nucleus, cytoskeleton, and extracellular matrix

It is important to view nuclear organization and structure in the context of the overall cellular architecture. Contrary to early text book drawings, the nucleus is not simply a large organelle floating in the cytoplasm, but is instead intricately connected to the surrounding cytoskeleton. Application of force to the extracellular surface or the cytoskeleton can result in substantial nuclear deformations (Maniotis et al. 1997, Caille et al. 1998) and expression of mechanosensitive genes (Chien et al. 1998, De Keulenaer et al. 2002). The exact manner of force transmission and the mechanism by which mechanical stimuli can trigger activation of specific genes remain largely unknown. However, evidence for a physical nucleo-cytoskeletal link is now emerging. Many of the major discoveries in this field were first made in C. elegans and Drosophila, and then subsequently found to be conserved in vertebrates. In the current model (see Warren et al. 2005, Stewart et al. 2007 for detailed reviews), nuclear lamins and other nuclear envelope proteins interact with SUN family proteins located at the inner nuclear membrane (Worman \& Gundersen 2006, Ketema et al. 2007, Liu et al. 2007, Wheeler et al. 2007). SUN proteins in turn associate across the luminal space with the KASH domain of nesprins residing on the outer nuclear membrane (Padmakumar et al. 2005, Crisp et al. 2006). Nesprins are encoded by at least three different genes and are found in many splicing variants. Nesprins are spectrin-repeat-containing proteins that are anchored to the outer nuclear membrane through their C-terminal KASH domain (Warren et al. 2005). Different nesprin subtypes contain $\mathrm{N}$-terminal cytoplasmic binding domains for direct interaction with specific cytoskeletal structures, e.g. actin fibres for the giant forms of nesprin-1 and -2 , or for plectin, an intermediate filament binding protein, in nesprin-3 (reviewed in Broers et al. 2006, Verstraeten et al. 2007) (Figure 1). The spectrin repeats found in repeats of nesprin could further contribute to interactions with cytoskeletal structures such as actin. Recent evidence suggests 


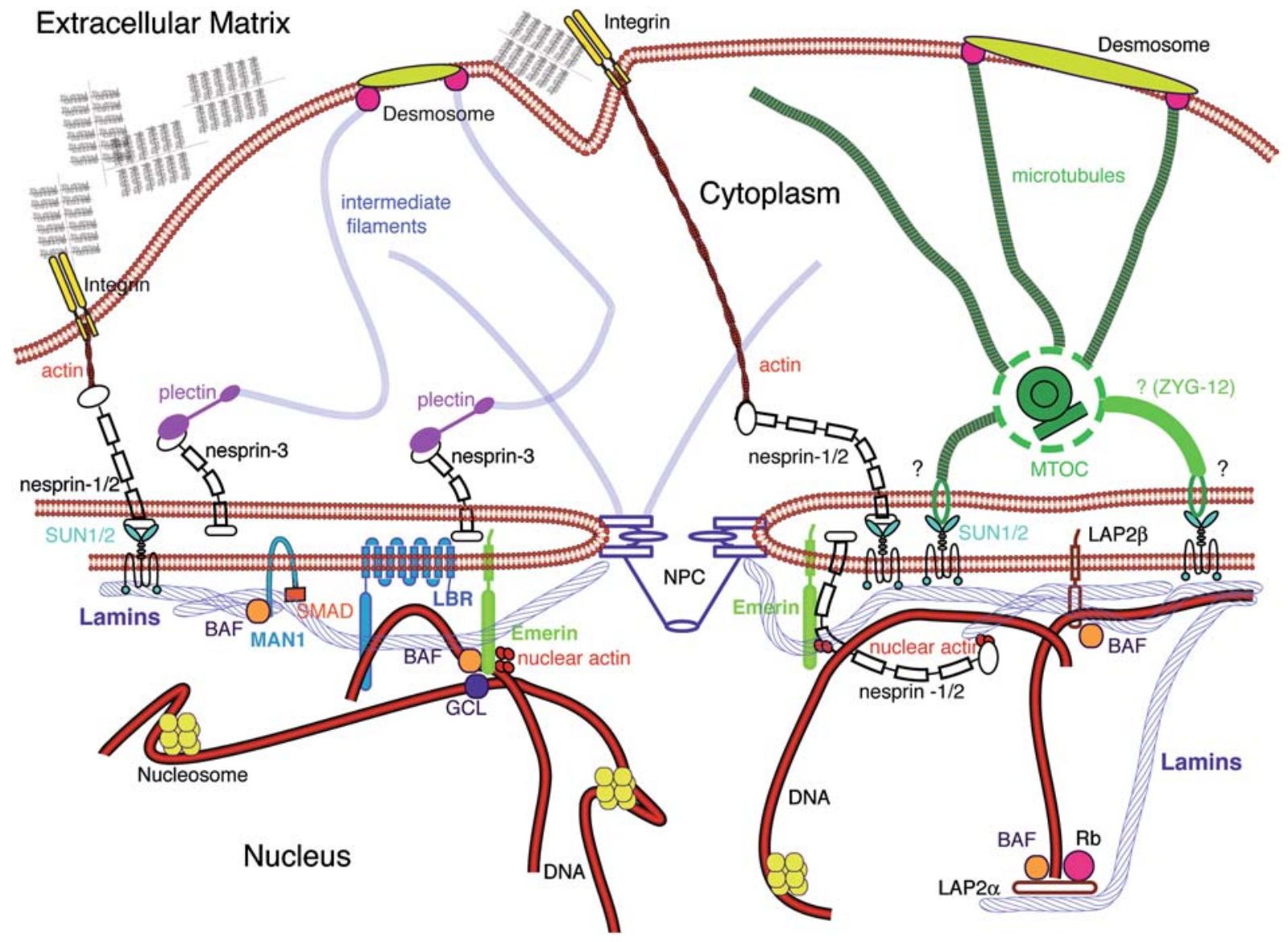

Figure 1. The physical connection between chromatin and extracellular matrix. Nuclear lamins polymerize into a filamentous network lining the inner nuclear membrane and spacing the nuclear pore complexes (NPC). Lamins interact with multiple nuclear envelope-associated proteins such as the lamin B receptor (LBR), the LEM-domain proteins such as LAP2 $\beta$, Emerin and MAN1, and SUN-proteins. LEM-proteins further interact with chromatin through BAF, whereas SUN-proteins link the nuclear envelope to the cytoskeleton by their interaction with the outer nuclear membrane-bound nesprins. The giant isoforms of nesprins- 1 and -2 contain an actin-binding domain, whereas nesprin- 3 mediates the interaction with intermediate filaments through plectin. Spectrin repeats characteristic of most nesprin isoforms might further interact with cytoskeletal proteins, especially actin. Actin binds to integrins at the plasma membrane, and thereby connects to the extracellular matrix. Intermediate filaments and possibly also microtubules can anchor the plasma membrane at the desmosome, which is a junctional complex specialized for cell-to-cell adhesion. Through their interaction with nuclear envelope proteins, nuclear lamins can also affect multiple transcription factors such as SMAD (through MAN1), GCL (through emerin), and pRb (through direct interactions and through LAP2 $\alpha$ ). (Figure adapted from Verstraeten et al. (2007) and reproduced with permission from Bentham Science Publishers Ltd.)

that emerin, another nuclear envelope protein that binds to lamin $\mathrm{A} / \mathrm{C}$, could participate in coupling microtubules and the centrosome to the nuclear envelope (Salpingidou et al. 2007). The cytoskeleton itself is physically connected to the extracellular matrix through integrin receptors and focal adhesions, completing the physical link between chromatin and the extracellular matrix. Because of this continuous physical connection from the nucleus to the extracellular environment, it is essential to complement studies on isolated nuclei with techniques that probe the nuclear mechanics in intact, living cells.

\section{Changes in nuclear mechanics throughout development and disease}

The physical connection between the nucleus, the cytoskeleton, and the extracellular matrix provides a potential mechanism through which extracellular forces and the mechanical properties of the cellular 
environment can affect nuclear shape and architecture and thereby mediate changes in genome expression and protein synthesis (Thomas et al. 2002). Cells grown on different physicochemical substrata that modulate cell adhesion and spreading, for example, display specific changes in nuclear architecture, each associated with distinct chromatin structure and gene expression patterns (Vergani et al. 2004, Engler et al. 2006). These mechanosensitive changes in gene expression could arise from changes in chromatin structure with alterations in the affinity of transcription factors for DNA or their transport to or within the nucleus, or from alterations in nuclear matrix organization and polymerase activity.

Alterations in the mechanical properties of the nucleus and chromatin seem essential for normal development and function. Micropipette aspiration of nuclei from cultured human embryonic stem cells revealed highly deformable nuclei that were up to 6-fold softer than nuclei from differentiated cells (Pajerowski et al. 2007). Furthermore, strain- or shear-stress-induced changes of the nucleus and chromatin structure are expected to play a physiologically important role in tissue homeostasis. For example, vascular smoothmuscle cells have the ability to transduce mechanical stimuli into intracellular signals that result in alterations of gene expression (Haga et al. 2007), and cardiac myocytes similarly respond to mechanical strain with expression of hypertrophic signals (Lammerding et al. 2004a).

Mutations in lamin $\mathrm{A} / \mathrm{C}$ result in multiple disease phenotypes, commonly referred to as laminopathies, ranging from cardiac or skeletal muscular dystrophy to premature ageing (reviewed in Stewart et al. 2007, Verstraeten et al. 2007). Aberrations in nuclear integrity and chromatin organization are thought to contribute to these phenotypes. In particular, nuclei bearing lamin $\mathrm{A} / \mathrm{C}$ mutations causing muscular dystrophy or Hutchinson-Gilford progeria syndrome (HGPS) show loss of peripheral heterochromatin. Furthermore, nuclei of HGPS patient fibroblasts were found to become increasingly stiffer over time (V.L.R.M. Verstraeten et al., unpublished), whereas lamin A/C-deficient mouse embryonic fibroblasts show reduced mechanical stiffness and increased fragility (Broers et al. 2004, Lammerding et al. 2004b). It remains uncertain to what extent the changes in nuclear stiffness result from alterations in the nuclear lamina or modifications in chromatin structure, prompting additional investigations.
Taken together, these examples highlight the relevance of nuclear and, in particular, chromatin mechanics in normal human development, cell function and disease. Here we will elaborate on the experimental techniques for study of nuclear mechanics in intact nuclei and living cells and the extent to which these techniques can be applied to the study of chromatin mechanics.

\section{Techniques}

A number of experimental techniques have been developed and applied to characterize the physical properties of the nucleus in intact cells. Each of these approaches has its own strengths and limitations. Some only probe the relative stiffness of the nucleus to the cytoskeleton or measure gross nuclear mechanical abnormalities, whereas others give a more precise evaluation of nuclear structure but require the isolation of nuclei or intrusive and disruptive treatment of cells. In the following, we introduce and discuss some of the techniques most commonly applied to probe the physical properties of the nucleus and chromatin in isolated nuclei and living cells.

\section{Micropipette aspiration experiments}

One of the first and most direct techniques to study the physical properties of intact nuclei is micropipette aspiration. This technique was pioneered by Evans and colleagues (Evans \& Hochmuth 1976) as a tool to study the viscoelastic properties of membranes and thin-shelled vesicles. Subsequently, it has been employed to study the mechanical behaviour of leukocytes (Chien et al. 1984), blood cells (Evans 1983 ) and various other cell types. For over 30 years now, micropipette aspiration of nuclei has been used to transplant nuclei from somatic cells into oocytes (Du Pasquier \& Wabl 1977). Recently, this technique has emerged as an important tool in the study of the physical properties of isolated nuclei (Guilak et al. 1999, Dahl et al. 2004, 2006, Deguchi et al. 2005) and nuclei within living cells (Rowat et al. 2005, 2006, Pajerowski et al. 2007).

In micropipette aspiration experiments, nuclei are partially aspirated into a micropipette with a 2-10 $\mu \mathrm{m}$ opening (see Figure 2A,B) by applying a precisely controlled aspiration pressure in the range 100-10 000 Pa (Lammerding et al. 2007). The applied pressure is measured by an inline 
A

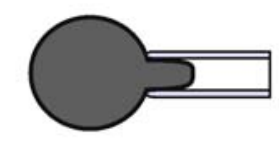

C

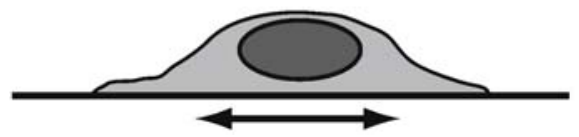

B

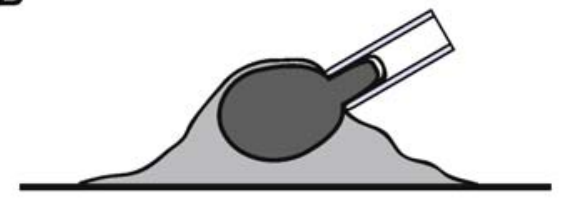

D

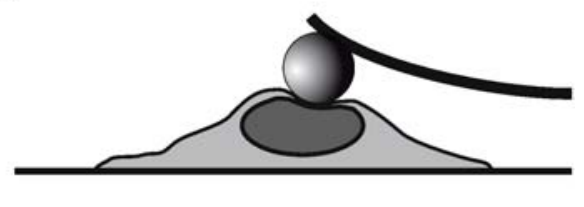

Figure 2. Experimental techniques to probe nuclear mechanics. (A) Micropipette aspiration of isolated nuclei. (B). Micropipette aspiration of nuclei in living cells. (C) Cellular strain experiments on adherent cells. (D) Atomic force microscopy on the nucleus in adherent cells.

pressure transducer, while induced nuclear deformations are monitored through a high-power objective on an inverted microscope. Nuclei can be isolated from cells by detergent treatment or by mechanical disruption of osmotically swollen cells with a Dounce homogenizer (Dahl et al. 2005). Isolated nuclei can be further purified by centrifugation through a sucrose gradient that removes excessive membranes from the endoplasmic reticulum or other kinds of cytoplasmic contamination (Dahl et al. 2005). Alternatively, micropipette aspiration can be performed on nuclei within living cells that have been treated with cytoskeleton-disrupting drugs such as latrunculin $\mathrm{A}$, which inhibits actin polymerization (Rowat et al. 2006, Pajerowski et al. 2007).

Several complementary analysis methods have been developed to convert the imaged nuclear deformation into quantitative measurements for comparison between cell types or treatment conditions. In the most common approach, the aspirated length of the nucleus (i.e. the tongue length within the micropipette) is normalized to the micropipette radius and the aspiration pressure. Assuming that the nucleus behaves as a homogeneous, isotropic and incompressible elastic medium with the volume outside the pipette resembling an infinite half-space (Dahl et al. 2005), the creep compliance of the nucleus can be calculated from these measurements as

$$
J(t)=\frac{2 \pi \Phi}{3} \frac{1}{P} \frac{\Delta L(t)}{R_{\mathrm{P}}}
$$

where $\Phi$ is a shape factor (here $\Phi=2.1$; Theret et al . 1998)], $P$ is the applied aspiration pressure, $\Delta L(t)$ is the aspirated length at time $t$, and $R_{\mathrm{P}}$ is the pipette radius.

Fluorescent labelling of nuclear components such as the nuclear lamina or nucleoli by fluorescent dyes or GFP-fusion proteins enables a more detailed study of deformations in these components by fluorescence/confocal imaged microdeformation (FIMD or CIMD). Analysing nuclei from GFP-lamin A transfected cells, Rowat and co-workers (Rowat et al. 2005,2006 ) showed that the decrease in fluorescence intensity towards the aspirated tip corresponds to aspiration-induced strain of the nuclear lamina that is consistent with the nonlinear, two-dimensional elasticity theory. In contrast to the elastic lamina, the nuclear membranes show more fluid-like behaviour during aspiration, and fluorescent labelling of the lipid membranes reveals persistent blebbing of the lipid membrane during aspiration (Rowat et al. 2006, Pajerowski et al. 2007). Observation of DNA labelled with SYTOX orange during micropipette aspiration indicates that the nuclear interior behaves as a soft, gel-like material that initially displays a substantial decrease in volume before becoming resistant to further deformation (Rowat et al. 2006). More detailed micropipette aspiration studies by Pajerowski and co-workers (Pajerowski et al. 2007) indicate that at short time scales, i.e. less than 10 seconds, nuclei show mostly reversible viscoelastic deformations but then undergo lasting, plastic deformations at longer time scales. These irreversible deformations include flow, distension and reorganization of the chromatin. GFP-histone 2B (GFPH2B)-labelled chromatin shows sheared deformation 
of the chromatin linked to the elastic nuclear lamina and reveals compaction of chromatin during aspiration. GFP-nucleophosmin (GFP-B23)-labelled nucleoli follow the 'flow' of chromatin but appear stiffer than the surrounding nucleoplasm. Interestingly, this group also observed stress-stiffening of the isolated nuclei, similar to the behaviour of extensional studies in individual chromatin fibres (Pajerowski et al. 2007). Taken together, these data suggest that while the elastic stiffness of the nuclear lamina plays an important role during the initial deformation, the physical properties of the chromatin determine the rheological behaviour of nuclei during micropipette aspiration for larger deformations. Interestingly, while some groups observe continued creep behaviour during micropipette aspiration, i.e. the nucleus continues to be aspirated into the pipette (Dahl et al. 2005, 2006, Pajerowski et al. 2007), others observe a viscoelastic response that quickly reaches an equilibrium without further deformation (Guilak et al. 1999, Deguchi et al. 2005, Rowat et al. 2005, 2006).

In addition to fluorescently labelling specific nuclear components, reversible swelling of isolated nuclei by varying the salt concentration of the aspiration buffer can be used to identify the contribution of both the lamina and nucleoplasm to nuclear deformation during micropipette aspiration. High concentrations of divalent salts result in condensed nuclei, whereas lower ionic strength buffers yield swollen nuclei that more closely resemble the nuclear shape observed in intact cells (Dahl et al. 2005). Studying osmotically swollen Xenopus oocytes in which the nuclear lamina became loose from the underlying chromatin, Dahl and co-workers (Dahl et al. 2004) found that the nuclear lamina represents an elastic network with an elastic modulus of $\sim 25 \mathrm{mN} / \mathrm{m}$ that is the main contributor to nuclear stiffness. However, Xenopus oocyte nuclei are significantly larger than nuclei from somatic cells, and their nuclear structure may not be representative of somatic interphase nuclei, so caution should be employed in interpreting these results. Nonetheless, similar findings were subsequently observed in green monkey kidney epithelial cells and human skin fibroblasts (Dahl et al. 2005, 2006). These studies suggest that, in condensed nuclei, chromatin serves as the primary force-bearing element, whereas the elastic properties of the nuclear lamina become increasingly important in swollen nuclei. It is noteworthy that swollen nuclei generally appear softer (Dahl et al. 2005, 2006); at 1 second of aspiration, condensed nuclei had an apparent elasticity of $5.72 \pm 2.15 \mathrm{kPa}$, while swollen nuclei had an apparent elasticity of $0.90 \pm 0.26 \mathrm{kPa}$. These and other studies confirm that nuclei are significantly stiffer than the surrounding cytoskeleton (Guilak et al. 1999, Dahl et al. 2005). Importantly, the specific physical properties can change with cell differentiation and in disease. Nuclei in human embryonic stem cells are initially highly deformable and become approximately 6-fold stiffer during terminal differentiation (Pajerowski et al. 2007). Knock-down of lamin $\mathrm{A} / \mathrm{C}$ in human epithelial cells results in a deformability similar to that of adult haematopoietic stem cells (Pajerowski et al. 2007). Thus, some of the observed differences could result from the absence of lamin $\mathrm{A} / \mathrm{C}$ in stem cells, as lamins $\mathrm{A}$ and $\mathrm{C}$ are the main contributors to nuclear stiffness (Lammerding et al. 2006), but could also reflect epigenetic differences in chromatin organization between stem cells and differentiated cells. In addition to changes in nuclear stiffness during differentiation and development, nuclear mechanics can also be altered by disease-causing mutations or mechanical stimulation. Micropipette aspiration experiments performed on fibroblasts from patients with Hutchinson-Gilford progeria syndrome revealed distinct changes in the mechanical properties of the nuclear lamina (Dahl et al. 2006), and lamin A/C-deficient mouse embryo fibroblasts have severely reduced nuclear stiffness (Lammerding et al. 2004b); persistent alterations in nuclear shape and increased nuclear stiffness have been observed in bovine aortic endothelial cells subjected to $24 \mathrm{~h}$ of shear stress (Deguchi et al. 2005).

These examples illustrate that micropipette aspiration can provide a powerful tool with which to study the physical properties of intact nuclei. The main limitations of this technique are that it requires isolation of nuclei or disruption of the cytoskeleton when performed on nuclei in living cells. Experimental results on isolated nuclei are extremely sensitive to variations in buffer composition and it is difficult to estimate the experimental conditions most closely resembling the cellular interior. Nuclear isolation by detergent treatment or mechanical disruption could also result in damage to the nuclei, especially when studying disease models with increased nuclear fragility, so caution should be taken to confirm the quality of the isolated nuclei. 
Furthermore, isolating nuclei or disrupting cytoskeletal structure to perform micropipette aspiration in intact cells significantly alters nuclear-cytoskeletal interactions and disturbs the normal mechanical environment of the cell. In adherent cells, the nucleus is in a pre-strained configuration, physically coupled to various cytoskeletal components, and disruption of these structures can result in collapse of the nuclear structure, leading to a more spheroidal and less spread appearance of the nucleus.

\section{Cellular strain experiments}

Another experimental approach to the study nuclear mechanics is to observe nuclear deformations in cells subjected to uniform substrate strain. Techniques to apply controlled uniaxial or biaxial strain to cultured cells were first developed to study the sensitivity of cells to mechanical stress (Banes et al. 1985). These strain experiments were subsequently combined with microscopic observations to probe subcellular mechanical properties (Barbee et al. 1994). Caille and co-workers applied uniaxial strain to bovine aortic endothelial cells to provide clear evidence for a mechanical coupling between the extracellular matrix and the nucleus (Caille et al. 1998). In our group, we have extensively used strain application to study the role of nuclear envelope proteins such as lamins and emerin (Figure 1) on the physical properties of the nucleus (Lammerding et al. 2004b, 2005). In these experiments, cells were plated on transparent silicone membrane dishes that were coated with fibronectin or other cellular matrix proteins to provide firm cell adhesion. Cells were serum-starved to minimize the fraction of cells in mitosis and focus on interphase nuclei, but serum starvation might not be necessary in slower-growing cultures or if the dependence of cell cycle phase on nuclear mechanics is of interest. Nuclei were labelled with a cell-permeable DNA-intercalating fluorescent dye (Hoechst 33342, Invitrogen, Carlsbad, CA, USA) and cells were subsequently kept in culture media or in Hanks buffered saline solution with calcium and magnesium. Silicone membrane dishes were mounted on a custom-built strain device and placed on an inverted fluorescence microscope. Phasecontrast images of the silicone membrane and fluorescent images of the nucleus were taken before, during and after strain application. At each stage, pictures of several fields of view can be taken to maximize the number of cells studied in a single experiment, but care should be taken not to strain cells for extended periods (more than 10 minutes) to minimize cell damage or potential cellular adaptation to the strain by cytoskeletal remodelling. In most of our experiments, we applied biaxial strain in the range 5-20\%, while other groups have applied uniaxial strain of more than $25 \%$ (Caille et al. 1998). Induced nuclear deformations increase approximately linearly with increased substrate strain (Caille et al. 1998, Lammerding et al. 2004b, Gilchrist et al. 2007), so higher strain generally results in larger nuclear deformations that can be better resolved, but excessive nuclear strain can cause cell damage and detachment and must thus be avoided. We recommend comparing phase-contrast images of pre- and post-strained cells to determine potential damage or detachment of cells during the experiments and to exclude affected cells from the analysis. Another useful marker for cell damage is the post-strain size of the nucleus. In intact cells the nucleus should approximately return to its initial size after strain application, while in partially detached or damaged cells the nucleus contracts significantly. Thus, cells which show marked (e.g. more than 1\%) decrease in nuclear size after strain application should be excluded from the analysis.

To control for variations in applied membrane strain, the induced nuclear strain is divided by the applied membrane strain to obtain the 'normalized nuclear strain' (Lammerding et al. 2004b) or 'strain transfer ratio' (Gilchrist et al. 2007). A value of 1 implies that the induced strain equals the applied substrate strain, while a ratio of 0 indicates a lack of strain-induced deformation. Note that values can exceed 1 when the deformations are larger than the applied strain; values can also fall in the negative range if the nucleus contracts during strain application. Typical values for the strain transfer ratio are $\sim 0.7-0.8$ for the cytoskeleton (Caille et al. 1998, Gilchrist et al. 2007) and $\sim 0.1-0.3$ for nuclei, which are stiffer than the surrounding cytoskeleton and therefore deform less (Caille et al. 1998, Lammerding et al. 2004b). The applied membrane strain is generally computed by tracking small particles (e.g. fluorescent beads) on the substrate itself, using pairs of matching control points identified in the pre-strain and full-strain images. The induced nuclear strain is determined from the images of the fluorescently labelled DNA. In mouse embryo fibroblasts, Hoechst 
33342-labelled nuclei show distinct chromocentres, consisting of dense heterochromatin, which can be easily tracked between pre-strain and full-strain images. The strain can then be computed by triangulation between sets of these control points or by minimizing the error of the linear affine image transformation required to map the pre-strain points to the locations observed during full strain. This technique can also be applied to subnuclear regions, allowing more detailed strain maps within the nucleus. Alternatively, one can use an imagecorrelation approach to calculate the induced nuclear strain. In this case, image transformation is applied to the base image (of pre-strained cells) and compared with the input image (of the same cells during strain application) by minimizing the normalized cross-correlation function (Lammerding et al. 2005, 2006). A similar approach with labelled texture correlation based on smaller image regions was recently described by Gilchrist and co-workers (Gilchrist et al. 2007).

The nuclear strain experiments offer the advantage that the physical properties of the nucleus are measured in situ, i.e. within its physiological environments and preserving the intricate intracellular structure. Furthermore, this approach avoids having to reconstitute buffers resembling the cellular interior, which is especially relevant as isolated nuclei are extremely sensitive to changes in divalent salt concentrations (Dahl et al. 2005). Another advantage of the cellular strain experiments is that many cells can be simultaneously strained, and the same technique can be used to study strain-induced gene induction and protein expression in larger cell populations. Most of our previous experiments have been focused on the role of the nuclear envelope in nuclear mechanics, but it is important to point out that the measured normalized nuclear strain reflects the mechanical properties of the entire nucleus, i.e. the nuclear envelope and the nucleoplasm, and also depends on the physical properties of the surrounding cytoskeleton. While the structure and physical properties of the CSK can be analysed by immunofluorescence and quantified by magnetic bead or particle tracking rheology (Lammerding et al. 2004b, Lee et al. 2007), the contribution of the individual nuclear components are more difficult to discern. Furthermore, in addition to probing the mechanical properties of the nucleus itself, the strain experiments also reveal information about the transmission of extracellular forces to the nucleus. While one of the biggest strengths, this characteristic is also the main limitation of these experiments, as the induced nuclear strain is a function not only of the physical properties of the nucleus but also of the surrounding cytoskeleton. Nonetheless, chromatin organization can be actively manipulated in these experiments through delivery of drugs that modify chromatin states such as trichostatin A (TSA) or microinjection of DNase into cells prior to the strain experiments.

\section{Indentation by $A F M$}

Atomic force microscopy (AFM) measurements typically probe the nucleus on a smaller length scale. AFM can follow the surface morphology at nanometer scale; it can measure intermolecular forces; and it can be used as a nanosurgical tool. No fixation is needed and imaging can be performed in physiological solutions. However, the greater the complexity of the biological structure, the lower the resolution achieved. Since an AFM tip, which typically consists of a cantilever with a pyramidal or spherical probe tip, must be pressed against the sample surface to assess the surface morphology, the plasticity of the underlying material determines the resolution. As such, a much higher resolution can be obtained when studying nuclear membrane proteins, e.g. nuclear pore complexes, on an isolated nuclear membrane patch spread on mica or glass compared to those on the total nucleus (Danker \& Oberleithner 2000). Forces measured are typically in the range of nanonewtons, whereas indentations are in the range of $50 \mathrm{~nm}$ to several micrometres. In general, AFM measurements of nuclear rheology are performed with isolated nuclei rather than intact cells. Isolated cell nuclei can be plated on rigid substrates that promote nuclear adhesion, (e.g. poly-L-lysine coating; Dahl et al. 2005), but are also feasible on substrates that minimize adhesion, such as poly-HEMA coated surfaces (Folkman \& Moscona 1978). Currently, most AFM studies have been performed on adherent cell nuclei, carefully balancing concentrations of poly-L-lysine that promote adhesion while avoiding nuclear aberrations due to excessive adhesion and surface interactions (Hategan et al. 2003). Depending on the type of experiment, different AFM probe tips can be used. The pyramidal probe tip has a very small radius of curvature, i.e. $25 \mathrm{~nm}$, whereas, the spherical micro-bead probe tip can have radii of 
$2.5 \mu \mathrm{m}$ and larger, which is ideal for probing the morphology of larger surface areas. Smaller tips provide a higher spatial resolution and measure local deformations at a very small length scale; the larger micro-bead probes allow larger deformations and appear more suitable for measuring the global mechanical properties of the nucleus. Similarly, a tip-less probe consisting of the flat surface of the cantilever can be used for nuclear compression experiments (described in Lammerding et al. 2007). For all of the probes, the applied force is determined by the stiffness of the cantilever and the deflection of the cantilever arm from the resting (zero-force) value. The indentation of the nucleus is measured by the vertical displacement of the cantilever from the point of contact with subtraction of the cantilever deflection. In general, there are two measuring modes - the contact (Domke et al. 2000) and the oscillating mode (Mahaffy et al. 2000). In the contact mode, the AFM probe is brought down onto the nucleus with a constant velocity, to cause a given degree of indentation. Here, results will depend on the rate and maximum depth of indentation. The difficulty of this mode, however, consists of an accurate determination of the point of contact. The oscillating mode is less sensitive to these errors, as the probe is brought down to an initial degree of indentation and then oscillates about that position while monitoring tip deflection and force. In experiments on intact nuclei, the contact mode is generally used, especially when using micro-bead probe tips.

AFM has been especially useful for the study of chromatin fibres. However, mainly partially unfolded chromatin fibres were studied owing to the tendency for chromatin condensation which coincides with loss of resolution at the level of nucleosomes (van Holde and Zlatanova 2007). Furthermore, this technique can also be implemented to study the mechanical properties of the nucleus and thus, indirectly, the mechanics of chromatin. Dahl and co-workers performed AFM on isolated nuclei on which more than 10 locations were typically probed per nucleus (Dahl et al. 2005). The results obtained were similar to those obtained with micropipette aspiration and indicated that nuclear deformability increases with time regardless of any characteristic timescale. The nucleus was found to be stiff, resisting distortion at short times, but softened and deformed more readily at longer times. Aside from its use for isolated nuclei, AFM may also be used to study nuclear mechanics in living adhering cells such as fibroblasts where the nucleus is positioned close to the cell membrane and therefore easily accessible to the AFM probe. However, the experimental interpretation in this case is complicated by the fact that the nucleus is surrounded by the cytoskeleton, and indentation studies thus reflect the effective stiffness of the nucleus and the surrounding cytoskeleton. Another limitation of AFM studies on intact nuclei is that they only probe the mechanical response of nuclei to compression, while many of the physiological mechanical stimuli are thought to exert tensile forces on the nucleus, especially considering the prestressed configuration of the cell (Wang et al. 2001).

\section{Particle-tracking microrheology}

Particle-tracking microrheology (PTM) has gained increasing popularity because (1) it has the highest spatial resolution, i.e. nanometers, among the methods probing cellular mechanics, (2) PTM causes only minor disturbance to the cell/nucleus, and (3) PTM provides a complete characterization of the elastic and viscous properties of the material. Single, double, and multiple particle-tracking methods have been used to define the rheological properties of the cytoskeleton and the nucleus. In particular, PTM studies have shown that the intranuclear region is much stiffer than the cytoplasm, and also more elastic than viscous, indicating that this region displays an unexpectedly strong solid-like behaviour (Tseng et al. 2004). The limitations are that particles have to be delivered into the cell/nucleus, which can be challenging with larger particles, and that PTM results can depend on the particle size and the mesh size of the probed cellular structure. As PTM is discussed in more detail elsewhere in this Chromosome Research review issue, we do not elaborate on this technique here.

\section{Outlook}

A number of exciting experimental techniques have been developed in recent years to quantify the nuclear mechanical properties of isolated nuclei and in living cells. However, the specific contributions of subnuclear components such as chromatin, nuclear bodies, and the nuclear envelope to the overall mechanical response of the nuclei under physiological 
conditions remain unclear, making it difficult to assess the consequences of local changes in structural chromatin organization on global nuclear properties. In the following, we present a brief overview of experimental approaches for actively manipulating chromatin structure to further elucidate the relationship between chromatin organization and nuclear mechanics.

Treatment of live cells with the histone deacetylase inhibitor trichostatin A (TSA) can be used as a simplified model for the open chromatin structure in stem cells. DNase treatment results in increased nuclear volume in isolated nuclei (Dahl et al. 2005) by decreasing chromatin density. Microinjection of DNase into the nuclei of living cells provides a simple tool for the study of the importance of DNA integrity to the physical properties of the nucleus. A more specific approach to modulating chromatin structure is the use of RNA interference (RNAi) directed against chromatin-modifying proteins such as histones or acetyl and methyl transferases. Such RNAi experiments can yield detailed information on the contribution of specific chromatin components or modifications to the overall structure and mechanics of the nucleus.

Aside from actively modulating chromatin structure with small-molecule drugs or genetic tools, fluorescent labelling procedures can be applied to investigate subnuclear mechanics. For instance, fluorescent labelling of individual chromosomes can be used to study the alterations of chromatin organization under cellular strain, e.g. differences between gene-poor chromosomes and gene-rich chromosomes or between chromosomes located at the nuclear periphery and the nuclear interior before and after strain application. One of the major challenges will be the use of these approaches in living cells, but several techniques such as microinjection or transfection of fluorescently labelled complementary DNA or RNA offer promise. From another perspective, fluorescently labelled proteins that are specific to nuclear compartments such as nucleoli or PMLbodies enable study of the physical behaviour of these proteins and yield information on the intranuclear coupling and relative stiffness of diverse nuclear components.

By combining the aforementioned techniques with emerging imaging technologies that probe transcriptional regulation and gene expression in living cells, a proper assessment of the interplay between structural changes of the nucleus and the induction of genes upon mechanical strain will be possible. This combination of technologies could putatively inform us about the long-sought cellular mechanosensors which are essential for mechanically induced gene expression, and provide new insights into the delicate relationship between chromatin structure and transcriptional activity in the living cell.

\section{Acknowledgements}

This work was supported by National Institutes of Health grants HL082792, NS059348, the American Heart Association grant 0635359N, and a research grant from the Progeria Research Foundation. Valerie Verstraeten is financially supported by a fellowship from the Netherlands Genomics Initiative and a fellowship from the 'Sint Annadal Stichting', Maastricht, the Netherlands.

\section{References}

Banes AJ, Gilbert J, Taylor D, Monbureau O (1985) A new vacuum-operated stress-providing instrument that applies static or variable duration cyclic tension or compression to cells in vitro. J Cell Sci 75: 35-42.

Barbee KA, Macarak EJ, Thibault LE (1994) Strain measurements in cultured vascular smooth muscle cells subjected to mechanical deformation. Ann Biomed Eng 22: 14-22.

Broers JL, Peeters EA, Kuijpers HJ et al. (2004) Decreased mechanical stiffness in LMNA-/- cells is caused by defective nucleo-cytoskeletal integrity: implications for the development of laminopathies. Hum Mol Genet 13: 2567-2580.

Broers JL, Ramaekers FC, Bonne G, Yaou RB, Hutchison CJ (2006) Nuclear lamins: laminopathies and their role in premature ageing. Physiol Rev 86: 967-1008.

Caille N, Tardy Y, Meister JJ (1998) Assessment of strain field in endothelial cells subjected to uniaxial deformation of their substrate. Ann Biomed Eng 26: 409-416.

Chien S, Schmid-Schonbein GW, Sung KL, Schmalzer EA, Skalak R (1984) Viscoelastic properties of leukocytes. Kroc Found Ser 16: 19-51.

Chien S, Li S, Shyy YJ (1998) Effects of mechanical forces on signal transduction and gene expression in endothelial cells. Hypertension 31: 162-169.

Crisp M, Liu Q, Roux K et al. (2006) Coupling of the nucleus and cytoplasm: role of the LINC complex. J Cell Biol 172: 41-53.

Dahl KN, Kahn SM, Wilson KL, Discher DE (2004) The nuclear envelope lamina network has elasticity and a compressibility limit suggestive of a molecular shock absorber. J Cell Sci 117: 4779-4786.

Dahl KN, Engler AJ, Pajerowski JD, Discher DE (2005) Powerlaw rheology of isolated nuclei with deformation mapping of nuclear substructures. Biophys J 89: 2855-2864. 
Dahl KN, Scaffidi P, Islam MF, Yodh AG, Wilson KL, Misteli T (2006) Distinct structural and mechanical properties of the nuclear lamina in Hutchinson-Gilford progeria syndrome. Proc Natl Acad Sci U S A 103: 10271-10276.

Danker T, Oberleithner H (2000) Nuclear pore function viewed with atomic force microscopy. Pflugers Arch 439: 671-681.

De Keulenaer GW, Wang Y, Feng Y et al. (2002) Identification of IEX-1 as a biomechanically controlled nuclear factor-kappaB target gene that inhibits cardiomyocyte hypertrophy. Circ Res 90: 690-696.

Deguchi S, Maeda K, Ohashi T, Sato M (2005) Flow-induced hardening of endothelial nucleus as an intracellular stressbearing organelle. J Biomech 38: 1751-1759.

Domke J, Dannohl S, Parak WJ, Muller O, Aicher WK, Radmacher M (2000) Substrate dependent differences in morphology and elasticity of living osteoblasts investigated by atomic force microscopy. Colloids Surf B Biointerfaces 19: 367-379.

Du Pasquier L, Wabl MR (1977) Transplantation of nuclei from lymphocytes of adult frogs into enucleated eggs: special focus on technical parameters. Differentiation 8: 9-19.

Engler AJ, Sen S, Sweeney HL, Discher DE (2006) Matrix elasticity directs stem cell lineage specification. Cell 126: 677-689.

Evans E (1983) Bending elastic modulus of red blood cell membrane derived from buckling instability in micropipet aspiration tests. Biophys $J$ 43: 27-30.

Evans EA, Hochmuth RM (1976) Membrane viscoelasticity. Biophys $J$ 16: 1-11.

Folkman J, Moscona A (1978) Role of cell shape in growth control. Nature 273: 345-349.

Gilchrist CL, Witvoet-Braam SW, Guilak F, Setton LA (2007) Measurement of intracellular strain on deformable substrates with texture correlation. J Biomech 40: 786-794.

Gotzmann J, Foisner R (1999) Lamins and lamin-binding proteins in functional chromatin organization. Crit Rev Eukaryot Gene Expr 9: 257-265.

Guilak F, Ting-Beall HP, Baer AE, Trickey WR, Erickson GR, Setton LA (1999) Viscoelastic properties of intervertebral disc cells. Identification of two biomechanically distinct cell populations. Spine 24: 2475-2483.

Haga JH, Li YS, Chien S (2007) Molecular basis of the effects of mechanical stretch on vascular smooth muscle cells. J Biomech 40: 947-960.

Hategan A, Law R, Kahn S, Discher DE (2003) Adhesively-tensed cell membranes: lysis kinetics and atomic force microscopy probing. Biophys J 85: 2746-2759.

Ketema M, Wilhelmsen K, Kuikman I, Janssen H, Hodzic D, Sonnenberg A (2007) Requirements for the localization of nesprin-3 at the nuclear envelope and its interaction with plectin. J Cell Sci 120: 3384-3394.

Laguri C, Gilquin B, Wolff N et al. (2001) Structural characterization of the LEM motif common to three human inner nuclear membrane proteins. Structure 9: 503-511.

Lammerding J, Kamm RD, Lee RT (2004a) Mechanotransduction in cardiac myocytes. Ann N Y Acad Sci 1015: 53-70.

Lammerding J, Schulze PC, Takahashi T et al. (2004b) Lamin A/C deficiency causes defective nuclear mechanics and mechanotransduction. J Clin Invest 113: 370-378.
Lammerding J, Hsiao J, Schulze PC, Kozlov S, Stewart CL, Lee RT (2005) Abnormal nuclear shape and impaired mechanotransduction in emerin-deficient cells. J Cell Biol 170: 781-791.

Lammerding J, Fong LG, Ji JY et al. (2006) Lamins A and C but not lamin B1 regulate nuclear mechanics. $J$ Biol Chem 281: 25768-25780.

Lammerding J, Dahl KN, Discher DE, Kamm RD (2007) Nuclear mechanics and methods. Methods Cell Biol 83: 269-294.

Lee JS, Hale CM, Panorchan P et al. (2007) Nuclear lamin A/C deficiency induces defects in cell mechanics, polarization, and migration. Biophys $J$ 93: 2542-2552.

Liu Q, Pante N, Misteli T et al. (2007) Functional association of Sun1 with nuclear pore complexes. J Cell Biol 178: 785-798.

Mahaffy RE, Shih CK, MacKintosh FC, Kas J (2000) Scanning probe-based frequency-dependent microrheology of polymer gels and biological cells. Phys Rev Lett 85: 880-883.

Malhas A, Lee CF, Sanders R, Saunders NJ, Vaux DJ (2007) Defects in lamin B1 expression or processing affect interphase chromosome position and gene expression. J Cell Biol 176: 593-603.

Maniotis AJ, Chen CS, Ingber DE (1997) Demonstration of mechanical connections between integrins, cytoskeletal filaments, and nucleoplasm that stabilize nuclear structure. Proc Natl Acad Sci U S A 94: 849-854.

Misteli T (2004) Spatial positioning; a new dimension in genome function. Cell 119: 153-156.

Padmakumar VC, Libotte T, Lu W et al. (2005) The inner nuclear membrane protein Sun 1 mediates the anchorage of Nesprin-2 to the nuclear envelope. J Cell Sci 118: 3419-3430.

Pajerowski JD, Dahl KN, Zhong FL, Sammak PJ, Discher DE (2007) Physical plasticity of the nucleus in stem cell differentiation. Proc Natl Acad Sci U S A 104: 15619-15624.

Parada L, Misteli T (2002) Chromosome positioning in the interphase nucleus. Trends Cell Biol 12: 425-432.

Pickersgill H, Kalverda B, de Wit E, Talhout W, Fornerod M, van Steensel B (2006) Characterization of the Drosophila melanogaster genome at the nuclear lamina. Nat Genet 38: 1005-1014.

Rowat A, Foster L, Nielsen M, Weiss M, Ipsen J (2005) Characterization of the elastic properties of the nuclear envelope. J R Soc Interface 2: 63-69.

Rowat AC, Lammerding J, Ipsen JH (2006) Mechanical properties of the cell nucleus and the effect of emerin deficiency. Biophys $J$ 91: 4649-4664.

Salpingidou G, Smertenko A, Hausmanowa-Petrucewicz I, Hussey PJ, Hutchison CJ (2007) A novel role for the nuclear membrane protein emerin in association of the centrosome to the outer nuclear membrane. J Cell Biol 178: 897-904.

Stewart CL, Roux KJ, Burke B (2007) Blurring the boundary: the nuclear envelope extends its reach. Science 318: 1408-1412.

Theret DP, Levesque MJ, Sato M, Nerem RM, Wheeler LT (1998) The application of a homogeneous half-space model in the analysis of endothelial cell micropipette measurements. J Biomech Eng 110: 190-199.

Thomas CH, Collier JH, Sfeir CS, Healy KE (2002) Engineering gene expression and protein synthesis by modulation of nuclear shape. Proc Natl Acad Sci U S A 99: 1972-1977.

Tseng Y, Lee JS, Kole TP, Jiang I, Wirtz D (2004) Microorganization and visco-elasticity of the interphase nucleus revealed by particle nanotracking. J Cell Sci 117: 2159-2167. 
van Holde K, Zlatanova J (2007) Chromatin fiber structure: Where is the problem now? Semin Cell Dev Biol 18: 651-658.

Vergani L, Grattarola M, Nicolini C (2004) Modifications of chromatin structure and gene expression following induced alterations of cellular shape. Int J Biochem Cell Biol 36: 1447-1461

Verstraeten VL, Broers JL, Ramaekers FC, van Steensel MA (2007) The nuclear envelope, a key structure in cellular integrity and gene expression. Curr Med Chem 14: 1231-1248.

Wang N, Naruse K, Stamenovic D et al. (2001) Mechanical behavior in living cells consistent with the tensegrity model. Proc Natl Acad Sci U S A 98: 7765-7770.
Warren DT, Zhang Q, Weissberg PL, Shanahan CM (2005) Nesprins: intracellular scaffolds that maintain cell architecture and coordinate cell function? Expert Rev Mol Med 7: 1-15.

Wheeler MA, Davies JD, Zhang Q et al. (2007) Distinct functional domains in nesprin-1alpha and nesprin-2beta bind directly to emerin and both interactions are disrupted in X-linked EmeryDreifuss muscular dystrophy. Exp Cell Res 313: 2845-2857.

Worman HJ, Evans CD, Blobel G (1990) The lamin B receptor of the nuclear envelope inner membrane: a polytopic protein with eight potential transmembrane domains. J Cell Biol 111: 1535-1542.

Worman HJ, Gundersen GG (2006) Here come the SUNs: a nucleocytoskeletal missing link. Trends Cell Biol 16: 67-69. 